OPEN ACCESS

Edited by:

Xinyi Liu,

University of Illinois at Chicago,

United States

Reviewed by:

Bo Han,

Capital Medical University, China

Qiong Wu,

The Chinese University of Hong Kong,

China

Guangyi Fan,

Beijing Genomics Institute (BGI),

China

*Correspondence:

Guolin Chen

guolinchen139@126.com

Specialty section

This article was submitted to

Computational Genomics,

a section of the journal

Frontiers in Genetics

Received: 15 July 2021

Accepted: 12 August 2021

Published: 09 September 2021

Citation:

Chen G, Zhang W and Ben Y (2021) Identification of Key Regulators

of Hepatitis C Virus-Induced

Hepatocellular Carcinoma by

Integrating Whole-Genome

and Transcriptome Sequencing Data.

Front. Genet. 12:741608.

doi: 10.3389/fgene.2021.741608

\section{Identification of Key Regulators of Hepatitis C Virus-Induced Hepatocellular Carcinoma by Integrating Whole-Genome and Transcriptome Sequencing Data}

\author{
Guolin Chen*, Wei Zhang and Yiran Ben \\ Department of Infectious Diseases, The First Affiliated Hospital of Harbin Medical University, Harbin, China
}

Background: Hepatitis C virus ( $\mathrm{HCV}$ ) infection is a major cause of cirrhosis and hepatocellular carcinoma (HCC). Despite recent advances in the understanding of the biological basis of HCC development, the molecular mechanisms underlying HCVinduced $\mathrm{HCC}(\mathrm{HCC}-\mathrm{HCV})$ remain unclear. The carcinogenic potential of HCV varies according to the genotype and mutation in its viral sequence. Moreover, regulatory pathways play important roles in many pathogenic processes. Therefore, identifying the pathways by which HCV induces HCC may enable improved HCC diagnosis and treatment.

Methods: We employed a systematic approach to identify an important regulatory module in the process of HCV-HCC development to find the important regulators. First, an HCV-related HCC subnetwork was constructed based on the gene expression in HCC-HCV patients and HCC patients. A priority algorithm was then used to extract the module from the subnetworks, and all the regulatory relationships of the core genes of the network were extracted. Integrating the significantly highly mutated genes involved in the HCC-HCV patients, core regulatory modules and key regulators related to disease prognosis and progression were identified.

Result: The key regulatory genes including EXO1, VCAN, KIT, and hsa-miR-200c-5p were found to play vital roles in HCV-HCC development. Based on the statistics analysis, EXO1, VCAN, and KIT mutations are potential biomarkers for HCV-HCC prognosis at the genomic level, whereas has-miR-200c-5P is a potential biomarker for HCV-HCC prognosis at the expression level.

Conclusion: We identified three significantly mutated genes and one differentially expressed miRNA, all related to HCC prognosis. As potential pathogenic factors of HCC, these genes and the miRNA could be new biomarkers for HCV-HCC diagnosis.

Keywords: genetic mutation, transcriptome, miRNA, hepatitis C. virus, hepatocellular carcinoma 


\section{INTRODUCTION}

Hepatocellular carcinoma (HCC) - the second leading cause of cancer-related deaths worldwide (Merte, 1989)-is often diagnosed at an advanced stage and progresses rapidly. Therefore, in HCC patients, early diagnosis is very important to improve their prognosis. Currently, early clinical screening methods for HCC involve serum alpha fetoprotein (AFP) detection and liver ultrasound examination (Sato et al., 1993). However, the sensitivity and specificity of markers such as AFP are marginal; moreover, ultrasound examination considerably relies on the subjective judgment of the operator, and conventional ultrasound results are often not useful for the conclusive identification of liver lesions. Therefore, a more effective, accurate method for screening liver cancer is needed urgently. As the understanding of cancer biology improves, liquid biopsy will become an increasingly useful tool for early diagnosis. Risk factors for HCC include cirrhosis, aflatoxin B intake, alcohol consumption, and hepatitis $B$ virus (HBV) and hepatitis $\mathrm{C}$ virus $(\mathrm{HCV})$ infection. Of these, HBV and HCV infections are the most notorious; in general, HBV- or HCV-positive patients have a 15-20-fold higher lifetime relative risk of HCC than HBV- and HCVnegative patients (El-Serag, 2012). To date, few studies have been focused on the factors leading to liver cancer in HCV patients. At present, HCV RNA, cirrhosis, and HCV genotype are thought to affect the occurrence of HCV-related liver cancer, but the involvement of these factors has not been conclusively proven. At present, the number of people affected by chronic HCV infection is 180 million-linked to $>350,000$ deaths annually (Li and Lo, 2015). Epidemiological studies have also shown that HCV is a risk factor for various diseases, including oral manifestations, glomerulopathies, type 2 diabetes mellitus, and insulin resistance (Montenegro et al., 2013; Carrozzo and Scally, 2014; Ozkok and Yildiz, 2014).

In total, $55-85 \%$ of people with HCV infection will develop chronic hepatitis C, and $20-30 \%$ of people with chronic liver disease will develop liver failure or cirrhosis (Mahale et al., 2017). Over the course of 30 years, $1-3 \%$ of patients with HCV without cirrhosis will develop HCC eventually (Huang et al., 2011; ElSerag, 2012). Moreover, one-third of HCC cases have been reported to be caused by hepatitis C (Parkin, 2006). At present, there are three major known mechanisms for HCV-induced HCC (HCV-HCC): direct pathways involving HCV core proteins, indirect pathways caused by oxidative stress and steatosis, and microRNA (miRNA)-related pathways (Tholey and Ahn, 2015). While biological signaling systems are complex, the analysis of linear pathways may still provide valuable insights (Weng et al., 1999). In the study of HCV, core genes have been found to be closely related to the carcinogenicity of chronic HCV infection. The expression of core genes has been experimentally shown to immortalize primary liver cells and induce cell transformation and carcinogenesis (Li et al., 2010). In addition, the genome sequencing analysis has demonstrated significant differences in the characteristics of liver cancer patients with or without HCV (Fishman et al., 2009). Taken together, these results indicate that core HCV gene mutations are closely associated with increased liver cancer risks.
In this study, the correlation between the key regulators and prognosis was investigated by integrating whole-genome and transcriptome sequencing data from The Cancer Genome Atlas (TCGA) and Gene Expression Omnibus (GEO) databases. We identified differentially expressed and mutated genes between HCV-HCC and HCC groups and performed functional enrichment analysis for genes in the module. Then, we explored the association of the key regulators with patient prognoses. The module and the key regulators may be potential biomarkers for predicting HCV-HCC.

\section{MATERIALS AND METHODS}

\section{The Cancer Genome Atlas and Gene Expression Omnibus Data Acquisition}

Gene mutation and mRNA and miRNA expression data as well as clinical information were downloaded from TCGA ${ }^{1}$ (Deng et al., 2016). In TCGA, liver hepatocellular carcinoma (LIHC) samples are divided into two groups: the first group contains HCV RNA or genotype or hepatitis C antibody in the patient's clinical information, and the other group does not; here, we named the two groups HCC-HCV and HCC. The gene/miRNA microarray as verifying cohorts GSE154211 (Wang et al., 2021) and GSE119159 (Umezu et al., 2020) were downloaded from GEO database. The data were normalized, and $\mathrm{R}$ and its packages were employed in all analysis steps.

\section{Differential Analysis}

MuTect2 Somatic Mutation data, analyzed using MuTect2, were download from TCGA. TCGA provides somatic mutation data in the MAF format. Therefore, we visualized somatic mutations using the R package "maftools" (Mayakonda et al., 2018). In total, 96 HCC-HCV samples and 269 HCC samples were present in the dataset. We calculated the mutational status of genes using the algorithm in maftools, and the genes with $p<0.05, \mathrm{OR}>2$, and number of mutations $>5$ were selected as the significantly and differentially mutated genes.

According to the groupings, we performed normalization and differential gene expression analysis using the R package "edgeR." False discovery rate (FDR) $<0.01$ and $\mid \log _{2}$ fold change (FC) $\mid$ $>1$ were used as cutoffs for identify differentially expressed genes (DEGs) for further analysis. Two R packages "pheatmap" and "ggplot2" were used for visualizing the heatmaps and volcano maps, respectively.

In total, $139 \mathrm{HCV}$ patients were enrolled in the GSE119159. They included 99 patients who had not developed HCC and 40 who had developed HCC. A total of 10 samples (tumor and non-tumor regions) from two HCV-related HCC patients and three HCC patients were used to find the gene candidates in HCV-related HCC in the GSE154211. For differential expression analysis, we used the R package "limma." $|\log 2 \mathrm{FC}|>1$ and $\operatorname{logFDR}<0.01$ were used as cutoffs to identify DEGs for further analysis.

\footnotetext{
${ }^{1}$ https://portal.gdc.cancer.gov/
} 


\section{Construction of the Transcription Factor-miRNA-mRNA Regulatory Network}

The human transcription factor (TF) and miRNA regulatory networks were constructed by integrating miRTarBase, TRANSFAC, and TransmiR (Vlachos et al., 2015;
Chou et al., 2018; Tong et al., 2019). The three databases include curated interactions among human TFs, miRNAs, and target genes. We uniformly named the genes and miRNAs within the regulatory networks according to the National Center for Biotechnology Information (NCBI) and miRbase databases. Moreover, all regulatory relationships within the regulatory

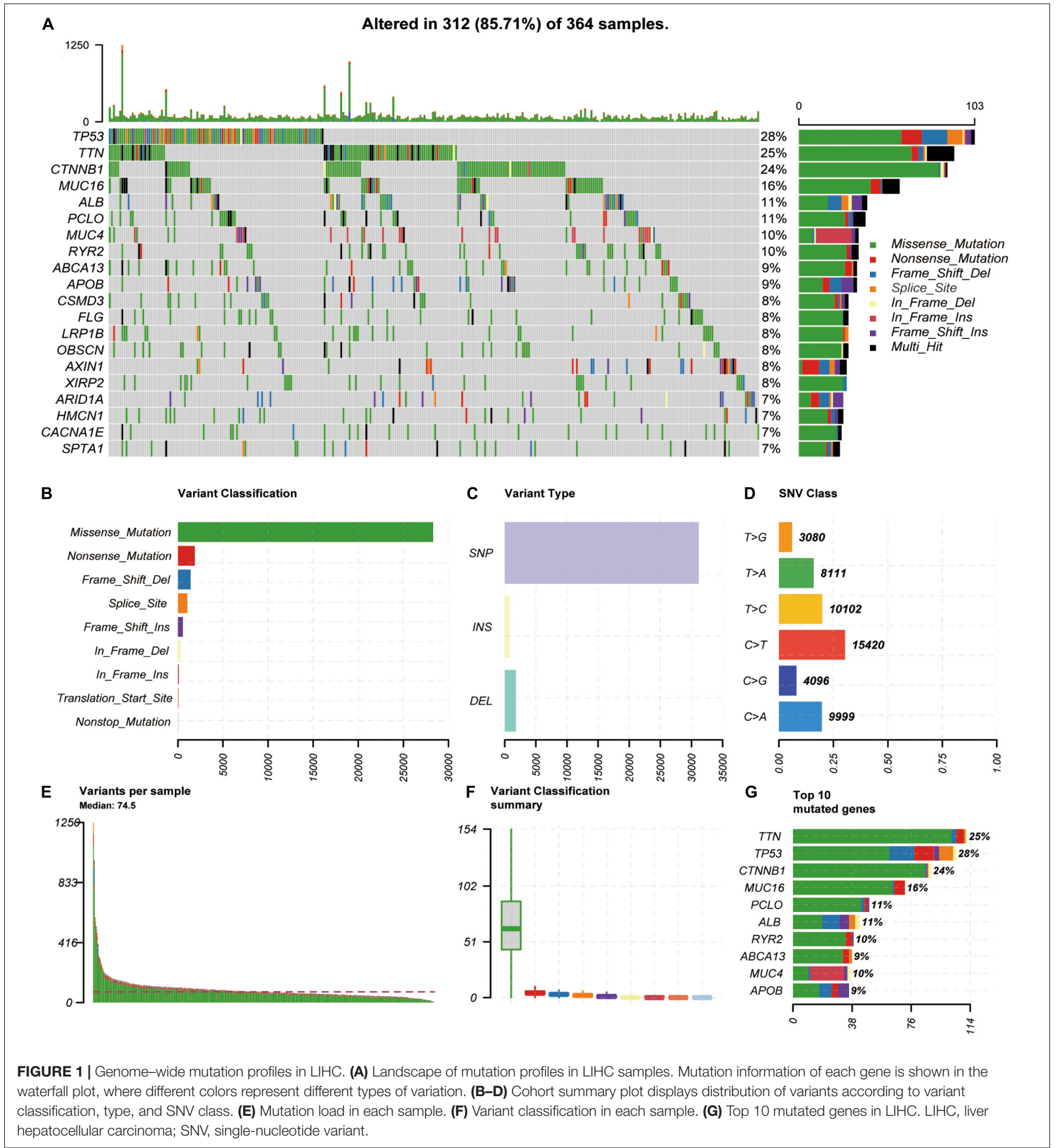


network were supported experimentally. In total, 888 TFs, 1,072 miRNAs, 3,150 target genes, and 18,056 edges were discovered in the regulatory network.

\section{Functional Enrichment Analysis}

The key regulatory gene symbols were converted to Entrez ID using the $\mathrm{R}$ package "org.Hs.eg.db." To identify the biological pathways involved in HCV-HCC occurrence and development, we employed Gene Ontology-biological process (GO-BP) function and Kyoto Encyclopedia of Genes and Genomes (KEGG) pathway enrichment analysis and visualized the results using the R packages "clusterProfiler" and "ggplot2."

\section{Survival Analysis}

We constructed an HCV-HCC-related subnetwork and identified key regulators from the subnetworks. Next, we investigated whether the key regulators could distinguish HCC patients with good or poor outcomes. From these data, we obtained TCGA HCC dataset with mRNA/miRNA expression and clinical information. Then, we used the key regulator expression values and mutation information to cluster all patients into two groups. The differential survival of the two study groups was finally assessed using the log-rank test.

\section{RESULTS}

\section{Mutation Analysis}

We downloaded and analyzed the somatic mutation data of 392 TCGA-LIHC samples. The mutation information of all genes in the samples is displayed as a waterfall diagram, with different colors representing different mutation types

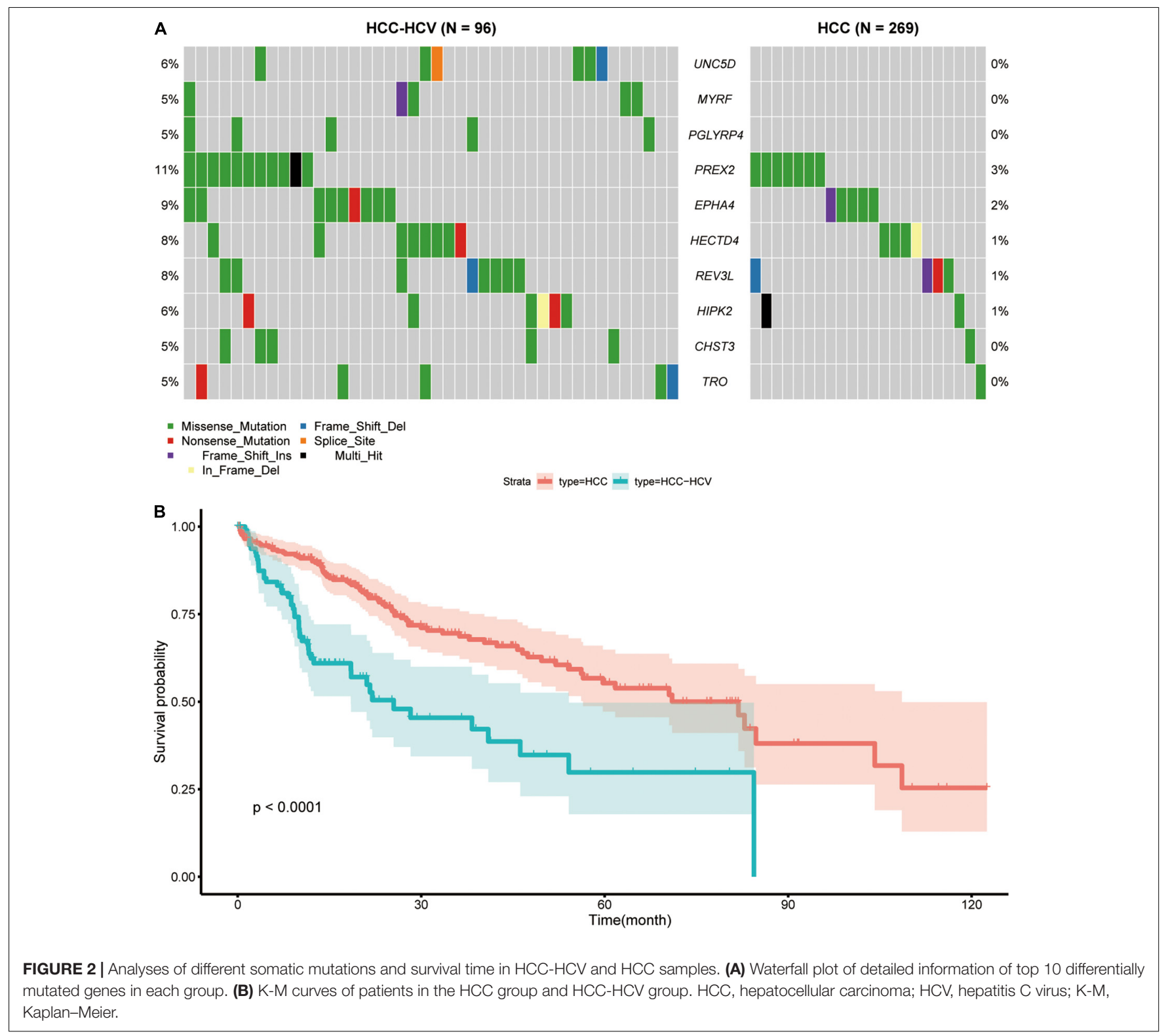


(Figure 1A). Further analysis showed that missense mutation, single-nucleotide polymorphisms (SNPs), and C > T accounted for the highest proportion of the variations (Figures 1B-D). The median number of variations in all HCC samples was 74.5 , and the maximum number of variations in a single sample was 1,250 (Figure 1E). The number of variations in different classifications in all samples is shown in a box diagram (Figure 1F). The top 10 mutated genes in the 392 samples were TTN (25\%), TP53 (28\%), CTNNB1 (24\%), MUC16 (16\%), PCLO (11\%), ALB (11\%), RYR2 (10\%), ABCA13 (9\%), MUC4 (10\%), and APOB (9\%; Figure 1G). In total, 96 HCC-HCV samples and 269 HCC samples were present in TCGA dataset; the survival analysis indicated that HCC patients without HCV lived significantly longer than HCC-HCV patients (Figure 2B). With the use of the maftools algorithm, 41 differentially mutated genes were identified (Supplementary Table 1). The top 10 differential mutated genes between the two groups of patients were UNC5D (6-0), MYRF (5-0), PGLYRP4 (5-0), PREX2 (117), EPHA4 (9-5), HECTD4 (8-4), REV3L (8-4), HIPK2 (6-2), CHST3 (5-1), and TRO (5-1; Figure 2A). Moreover, HCC-HCV patients with mutations in some genes had a poor prognosis (Supplementary Figure 1).

\section{Transcriptome Analysis}

Differentially expressed mRNAs and miRNAs were identified from two raw datasets: one containing GSE154211 and GSE119159 downloaded from the GEO database and another dataset from TCGA database. In total, $530 \mathrm{mRNA}$ and $30 \mathrm{miRNA}$ transcripts were observed to be expressed differentially in the HCC-HCV samples compared with HCC samples in TCGA dataset-including, respectively, 412 and 25 upregulated and 118 and five downregulated transcripts. Hierarchical clustering showed systematic variations in mRNA and miRNA expression in the HCC-HCV and HCC samples (Supplementary Figure 2). To identify the genes related to HCC-HCV in GSE154211, we first divided the expression data into four groups to identify DEGs between (A) HCC vs. HCC-HCV-adjacent, suggesting related to HCV-related carcinogenesis; (B) HCC-HCV vs. HCC, suggesting related HCV-related hepatocarcinogenesis; (C) HCCHCV-adjacent vs. HCC-adjacent, suggesting related to HCVrelated non-oncogenic effects; and (D) HCC vs. HCC-adjacent, suggesting related to non-HCV-related carcinogenesis. Four groups of data were then analyzed. Consequently, we identified 1,494 DEGs belonging to group A or B, but not group $\mathrm{C}$ or $\mathrm{D}$, as genes with strong potential to be relevant to

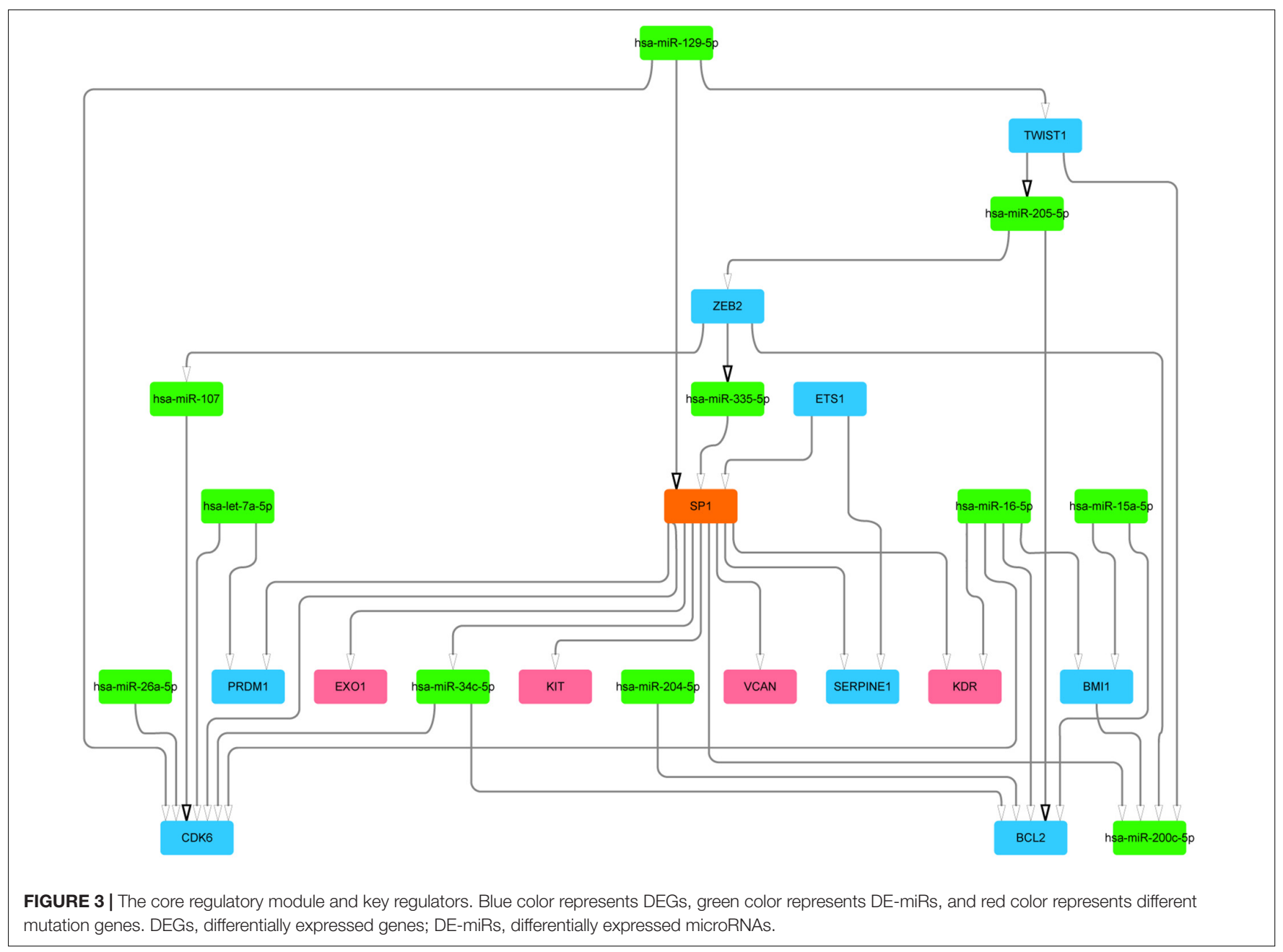


HCC-HCV (Supplementary Figure 3). In addition, 21 miRNA transcripts were observed to be differentially expressed in the developed HCC samples compared with the non-developed HCC samples in GSE119159, including nine upregulated and 12 downregulated transcripts.

\section{The Core Regulatory Module and Key Regulators}

To mine HCV-HCC-related regulatory relationships, we first constructed a TF-miRNA-mRNA regulatory network as a background network. Then, the HCV-induced HCC-related subnetwork was constructed by mapping DEGs into the background network. The nodes in the subnetwork contained DEGs and genes directly connected to the DEGs. In total, 359 TFs, 395 miRNAs, 739 target genes, and 2626 edges were present in the subnetwork.

We next mined the core regulatory module from the subnetwork by extracting the top 20 nodes ranked by closeness centrality and the edges among them. Notably, the regulatory relationships between these 20 nodes and differential mutated genes were added into the core regulatory module (Figure 3 and Supplementary Table 2). Finally, the core regulatory module contained 24 nodes and 36 edges.

To analyze the function of genes in the module, we conducted enrichment analysis of GO and KEGG, with FDR $<0.05$ used as the cutoff to identify statistically significant GO terms and KEGG pathways. We found that many GO terms and KEGG pathways were implicated in the HCV-HCC processes in previous studies. As shown in Figure 4A and Supplementary Table 3, in the biological process and molecular function categories, the significantly enriched genes were for vasculature development regulation (Vescovo et al., 2016), ameboidal-type and epithelial cell migration (Khera et al., 2017), cell aging (Naggie, 2017), cell-matrix adhesion (Ninio et al., 2019), and melanocyte differentiation and angiogenesis involved in wound healing (Mohsen et al., 2014). Furthermore, KEGG pathway analysis showed that the significantly enriched genes were for small cell lung cancer, miRNAs in cancer, PI3K-Akt signaling pathway (Cheng et al., 2015), Ras and p53 signaling pathways (Vescovo et al., 2016), cellular senescence (Shiu et al., 2017), endocrine resistance, and advanced glycation end products (AGE)-receptor for AGE (RAGE) signaling pathway in diabetic complications (Hyogo and Yamagishi, 2008; Figure 4B and Supplementary Table 4).

We further analyzed the genes in the core regulatory module and found that expression of EXO1, VCAN, has-miR-200c-5p, BMI1, has-miR-204-5p, and KIT was significantly correlated with HCC prognosis in all patients; and thus, these genes were considered key regulators (Figure 5A). In particular, we found that the patients with low EXO1, VCAN, or KIT expression had an adverse outcome $(H R<1$; Figure 5A). The HCC-HCV patients with mutations in these three genes have possibly also poor prognoses (Figures 5B-D). They may be potential biomarkers to predict the prognosis of patients at the genomic level. Moreover, we found that has-miR-200c-5P was significantly overexpressed in HCC-HCV samples (Figure 6A). The survival time of patients with high has-miR-200c-5P expression was significantly lower than that of patients with low expression (Figure 6B), suggesting that has-miR-200c-5P may be a potential biomarker to predict the prognosis of patients at the expression level.

Mutations in specific locations in EXO1 have been reported to inactivate proteins that increase cancer susceptibility (Welchew et al., 2002). KDR was also a significantly differential mutated gene in the module. KDR is the principal receptor that promotes the proangiogenic action of vascular endothelial growth factor
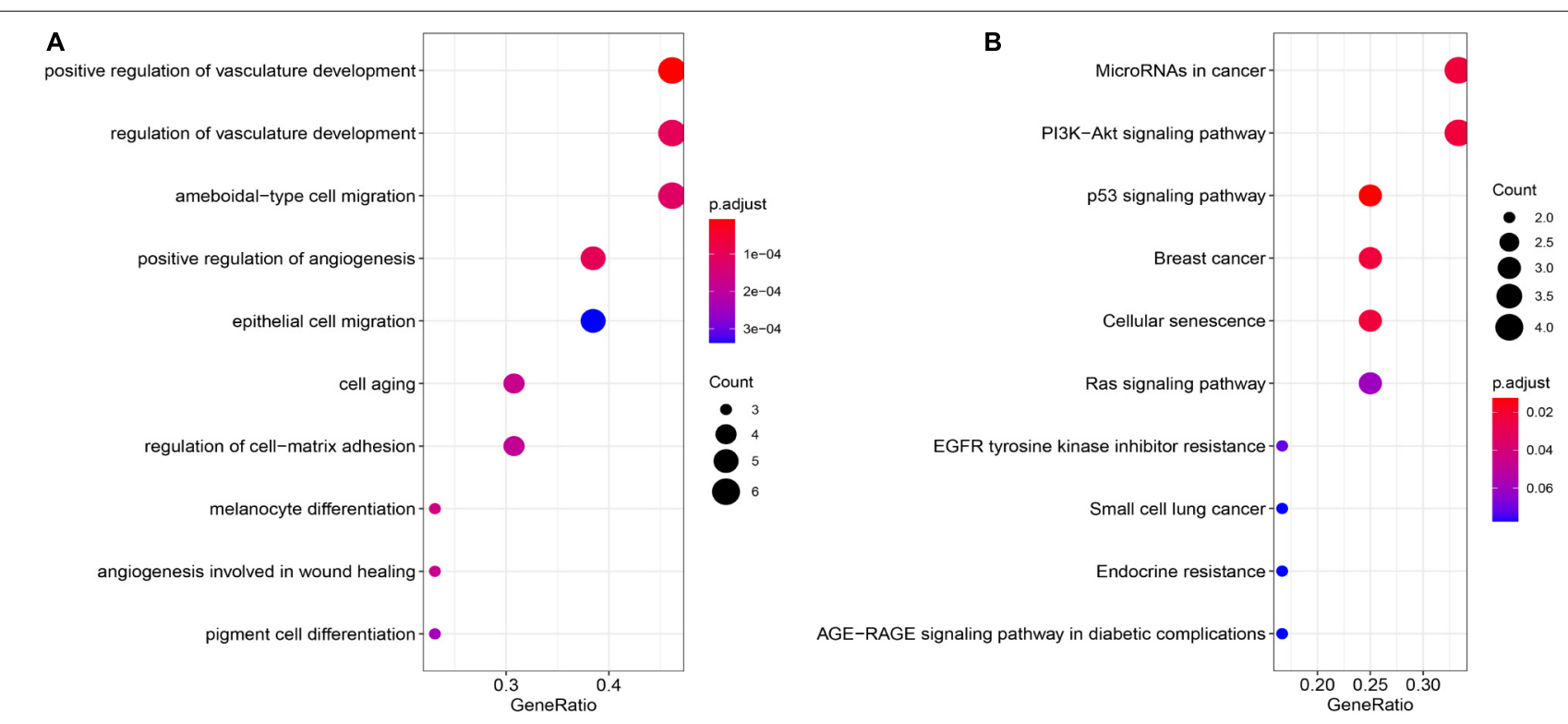

FIGURE 4 | GO and KEGG pathway enrichment analyses. (A) GO enrichment analysis of the module genes. (B) KEGG pathway enrichment analysis of the module genes. GO, Gene Ontology; KEGG, Kyoto Encyclopedia of Genes and Genomes. 
A

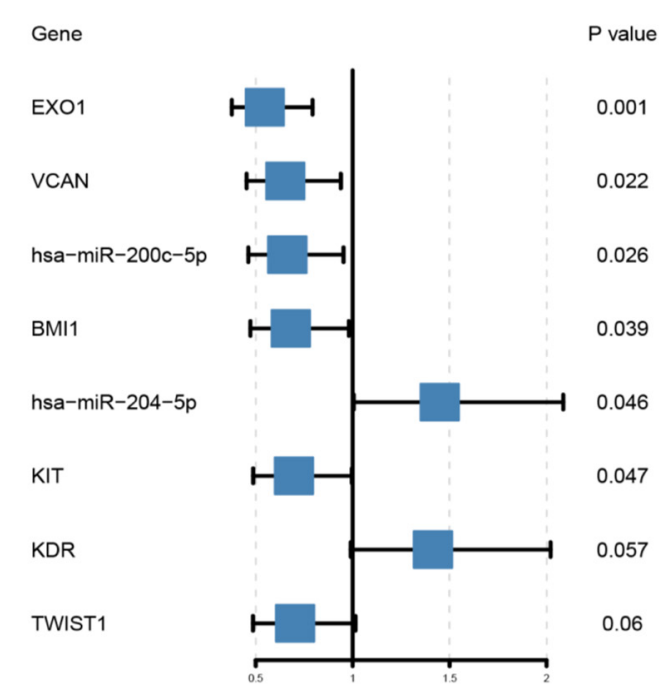

C

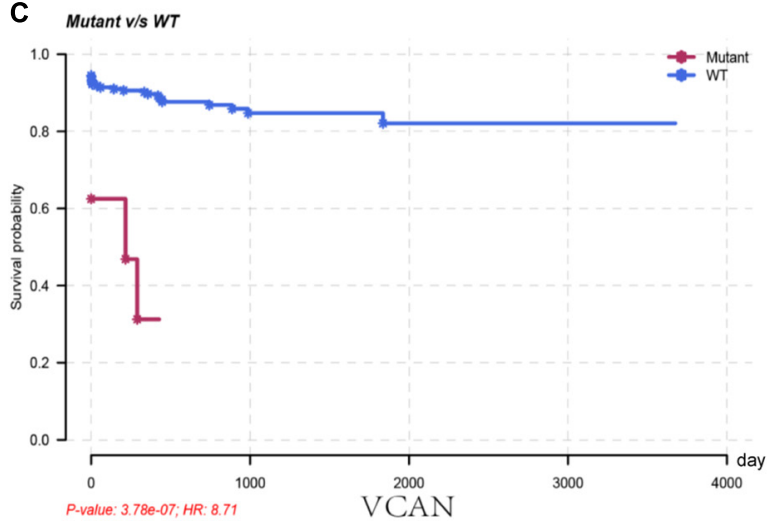

B

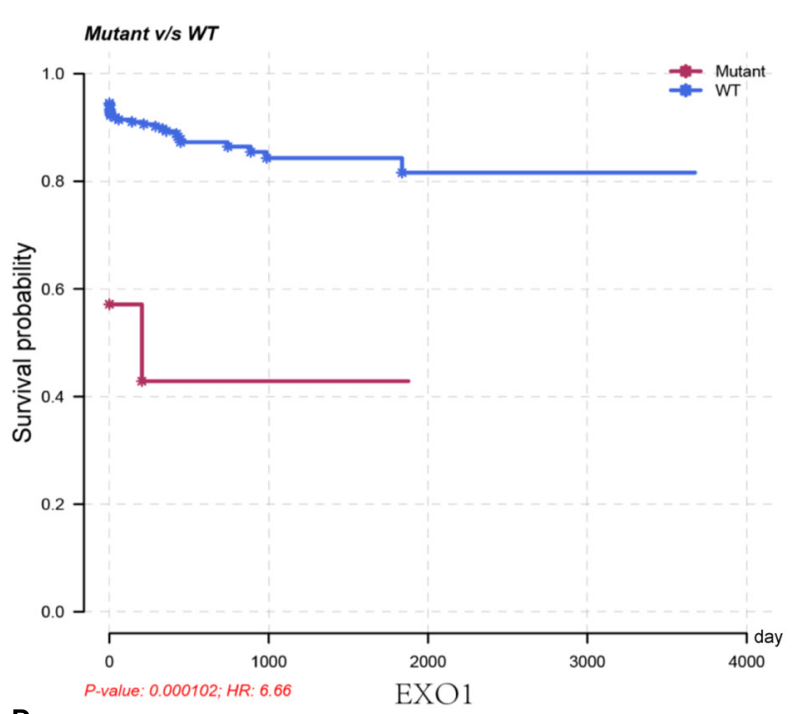

D

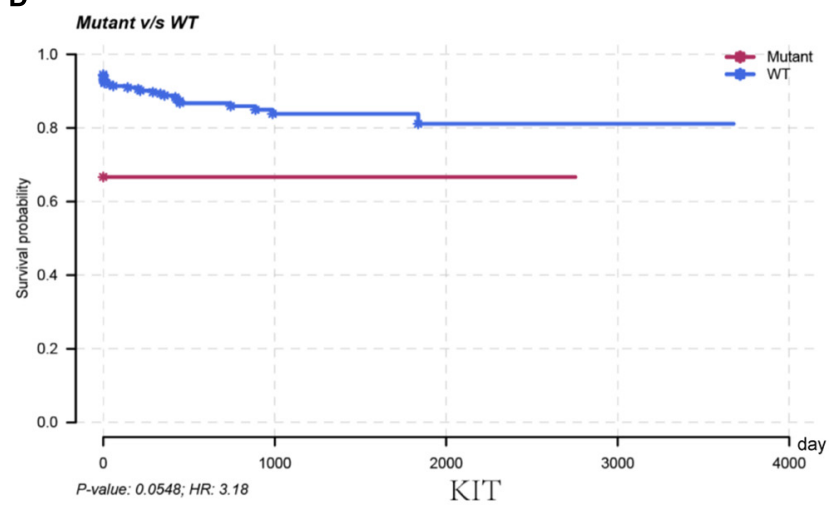

FIGURE $\mathbf{5}$ | Key regulators are related to worse survival rate. (A) Forest plot of hazard ratios showing the prognostic values of genes. (B-D) Survival curves of key regulators in the LIHC patients from TCGA dataset. LIHC, liver hepatocellular carcinoma; TCGA, The Cancer Genome Atlas.

and is involved in the tumorigenesis and progression of many malignancies, including HCC (Zheng et al., 2014). Moreover, $B C L 2$ was the downstream gene in the core regulatory module. $B C L 2$ can be functionally divided into antiapoptotic and proapoptotic groups. The balance between these two groups may determine the fate of a tumor cell. In HCC, this balance is often tilted toward the antiapoptotic members, leading to resistance to death and rapid proliferation in cancer cells (Alenzi et al., 2010). BCL2 expression in the HCC-HCV samples was lower than that in the HCC samples, but the difference was nonsignificant in TCGA data-which may be a reason for the worse prognosis of HCC-HCV.

\section{DISCUSSION}

HCC is responsible for the second highest global mortality rate, and HCV infection is a leading HCC risk factor. However, the mechanisms of HCC initiation, development, and metastasis are too complicated and thus unclear (Kanda et al., 2019). Currently, several factors are believed to influence the evolution of HCC from HCV infection. However, due to the lack of appropriate models or data, determining the specific role of $\mathrm{HCV}$ in the malignant transformation of liver cells is difficult. To identify and characterize these mechanisms, researchers have conducted genomic, transcriptomic, and epigenomic studies (Khatun and Ray, 2019).

Driver mutations in cancer-associated genes alter downstream signaling and transcription patterns, which are critical in cancer progression (Lai and Yang, 2013; Zhang et al., 2014, 2015; Huh et al., 2019). These studies have revealed that downstream gene mutations and gene expression changes are critical in hepatitis-induced liver cancer development. In this study, we found that mutations in a single gene can have a significant impact on disease prognosis in patients, whereas a combination of mutations in multiple genes is not an effective predictor of prognosis. This may be due to the low probability of simultaneous mutations of multiple genes; this 
A has-miR-200c-5p 审 HCC-HCV 审 HCC

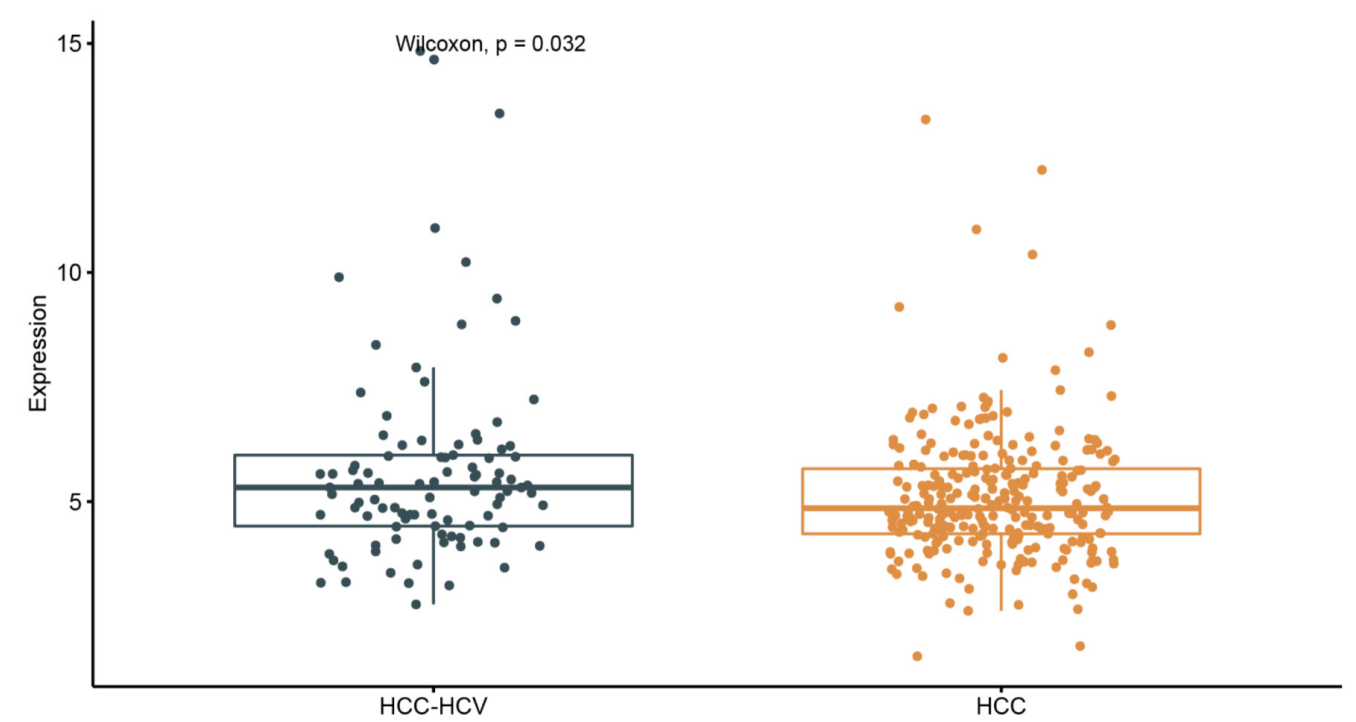

B

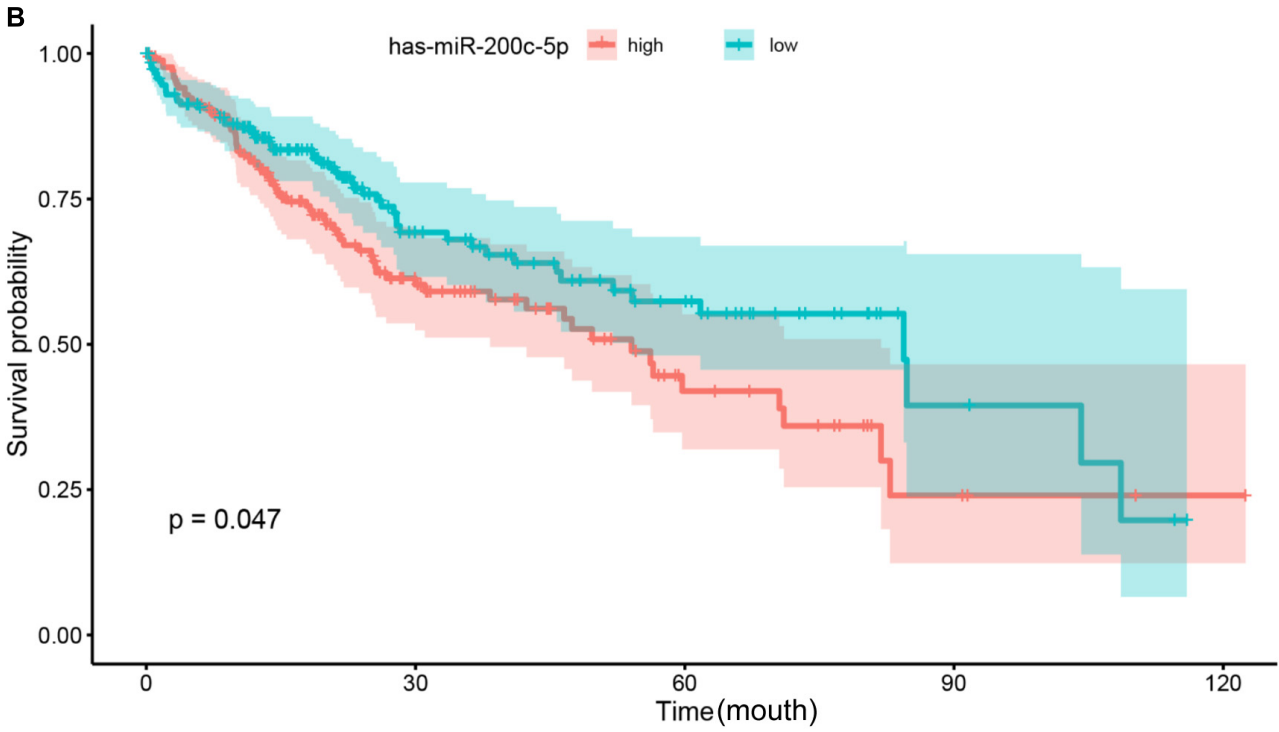

FIGURE 6 | Relationship between miR-200c expression level and patient prognosis. (A) Significant difference was found in the miR-200c expression between HCC-HCV and HCC patients. (B) HCC patients with high miR-200c expression have a relatively poor prognosis. HCC, hepatocellular carcinoma; HCV, hepatitis C virus.

will be studied further in our future work. Genomic research has found that long-term interactions between hepatitis virus and immune system causes significant stress and damage to the liver cells, making them undergo pathological adaptationeven after elimination of the virus. Non-coding RNA (ncRNA)related analysis has indicated that miRNAs play a crucial role in the posttranscriptional regulation of gene expression (Wong et al., 2018). Deregulation of certain miRNAs leads to the inactivation of tumor-suppressor genes and activation of HCC-related oncogenes. In this study, we incorporated whole-genome and transcriptomic sequence data to identify key regulators of HCV-HCC and found that abnormal expression of certain genes and miRNAs predict whether a patient with $\mathrm{HCV}$ infection will develop HCC. These genes may be potential biomarkers, which could enable HCC detection at significantly earlier stages.

In the functional enrichment analysis, we found that genes in the module were significantly enriched in the PI3K-Akt signaling pathway that promotes survival and growth in response to extracellular signals. KIT is an important receptor tyrosine kinase (RTK) that can stimulate the PI3K-Akt signaling pathway (Zhou et al., 2011). In addition, recent studies have shown that KIT exon 9 had a mutation resistant to TGF $\beta$, which can promote HCC development in HCV patients (El-Houseini et al., 2019). The miR-200 family - the most common family of miRNAs-demonstrates low expression in various cancers 
and is closely associated with tumorigenesis and outcome, particularly in HCC (Mao et al., 2020). has-miR-200c-5P is significantly overexpressed in $\mathrm{HCV}$ patients and promotes hepatic fibrosis (Ramachandran et al., 2013)—consistent with our results. Moreover, the survival time of patients with high has-miR-200c-5P expression was significantly lower than that of patients with low expression in the current study. In general, has-miR-200c-5P overexpression in esophageal cancer increases resistance to chemotherapeutic drugs by dysregulating PI3KAkt signaling pathway (Karakatsanis et al., 2013). Therefore, we speculate that has-miR-200c-5P and KIT may jointly regulate the PI3K-Akt signaling pathway and affect drug response and prognosis in HCV-HCC patients.

Although we identified some important regulatory genes and miRNAs, the specific underlying mechanisms could not be elaborated. Furthermore, HCC is complicated and multifactorial, and taking all factors into consideration was difficult. Therefore, additional studies determining whether genes correlated with $\mathrm{HCV}$-induced cancer are also correlated with liver cancer caused by other factors are warranted.

\section{REFERENCES}

Alenzi, F. Q., El-Nashar, E. M., Al-Ghamdi, S. S., Abbas, M. Y., Hamad, A. M., ElSaeed, O. M., et al. (2010). Original article: investigation of Bcl-2 and PCNA in hepatocellular carcinoma: relation to chronic HCV. J. Egypt. Natl. Canc. Inst. $22,87-94$.

Carrozzo, M., and Scally, K. (2014). Oral manifestations of hepatitis C virus infection. World J. Gastroenterol. 20, 7534-7543. doi: 10.3748/wjg.v20.i24.7534

Cheng, D., Zhang, L., Yang, G., Zhao, L., Peng, F., Tian, Y., et al. (2015). Hepatitis $\mathrm{C}$ virus NS5A drives a PTEN-PI3K/Akt feedback loop to support cell survival. Liver Int. 35, 1682-1691. doi: 10.1111/liv.12733

Chou, C. H., Shrestha, S., Yang, C. D., Chang, N. W., Lin, Y. L., Liao, K. W., et al. (2018). miRTarBase update 2018: a resource for experimentally validated microRNA-target interactions. Nucleic Acids Res. 46, D296-D302. doi: 10.1093/ nar/gkx1067

Deng, M., Bragelmann, J., Schultze, J. L., and Perner, S. (2016). Web-TCGA: an online platform for integrated analysis of molecular cancer data sets. BMC Bioinformatics 17:72. doi: 10.1186/s12859-016-0917-9

El-Houseini, M. E., Ismail, A., Abdelaal, A. A., El-Habashy, A. H., Abdallah, Z. F., Mohamed, M. Z., et al. (2019). Role of TGF-betal and C-Kit mutations in the development of hepatocellular carcinoma in hepatitis $\mathrm{C}$ virus-infected patients: in vitro study. Biochemistry 84, 941-953. doi: 10.1134/S000629791908 0108

El-Serag, H. B. (2012). Epidemiology of viral hepatitis and hepatocellular carcinoma. Gastroenterology 142, 1264-1273.e1. doi: 10.1053/j.gastro.2011.12. 061

Fishman, S. L., Factor, S. H., Balestrieri, C., Fan, X., Dibisceglie, A. M., Desai, S. M., et al. (2009). Mutations in the hepatitis $C$ virus core gene are associated with advanced liver disease and hepatocellular carcinoma. Clin. Cancer Res. 15, 3205-3213. doi: 10.1158/1078-0432.CCR-08-2418

Huang, Y. T., Jen, C. L., Yang, H. I., Lee, M. H., Su, J., Lu, S. N., et al. (2011). Lifetime risk and sex difference of hepatocellular carcinoma among patients with chronic hepatitis B and C. J. Clin. Oncol. 29, 3643-3650. doi: 10.1200/JCO.2011.36.2335

Huh, H. D., Kim, D. H., Jeong, H. S., and Park, H. W. (2019). Regulation of TEAD transcription factors in cancer biology. Cells 8:600. doi: 10.3390/cells 8060600

Hyogo, H., and Yamagishi, S. (2008). Advanced glycation end products (AGEs) and their involvement in liver disease. Curr. Pharm. Des. 14, 969-972. doi: $10.2174 / 138161208784139701$

Kanda, T., Goto, T., Hirotsu, Y., Moriyama, M., and Omata, M. (2019). Molecular mechanisms driving progression of liver cirrhosis towards hepatocellular carcinoma in chronic hepatitis B and C infections: a review. Int. J. Mol. Sci. 20:1358. doi: 10.3390/ijms20061358

\section{DATA AVAILABILITY STATEMENT}

The original contributions presented in the study are included in the article/ Supplementary Material, further inquiries can be directed to the corresponding author/s.

\section{AUTHOR CONTRIBUTIONS}

GC: study design, manuscript writing, and data analysis. WZ: data analysis, data collection, and manuscript writing. YB: data analysis and data collection. All authors have read, edited and approved of the final version of the manuscript.

\section{SUPPLEMENTARY MATERIAL}

The Supplementary Material for this article can be found online at: https://www.frontiersin.org/articles/10.3389/fgene. 2021.741608/full\#supplementary-material

Karakatsanis, A., Papaconstantinou, I., Gazouli, M., Lyberopoulou, A. Polymeneas, G., and Voros, D. (2013). Expression of microRNAs, miR21, miR-31, miR-122, miR-145, miR-146a, miR-200c, miR-221, miR-222, and miR-223 in patients with hepatocellular carcinoma or intrahepatic cholangiocarcinoma and its prognostic significance. Mol. Carcinog. 52, 297-303. doi: 10.1002/mc.21864

Khatun, M., and Ray, R. B. (2019). Mechanisms underlying hepatitis C virusassociated hepatic fibrosis. Cells 8:1249. doi: 10.3390/cells8101249

Khera, L., Paul, C., and Kaul, R. (2017). Hepatitis C Virus E1 protein promotes cell migration and invasion by modulating cellular metastasis suppressor Nm23H1. Virology 506, 110-120. doi: 10.1016/j.virol.2017.03.014

Lai, D., and Yang, X. (2013). BMP4 is a novel transcriptional target and mediator of mammary cell migration downstream of the Hippo pathway component TAZ. Cell. Signal. 25, 1720-1728. doi: 10.1016/j.cellsig.2013.05.002

Li, H. C., and Lo, S. Y. (2015). Hepatitis C virus: virology, diagnosis and treatment. World J. Hepatol. 7, 1377-1389. doi: 10.4254/wjh.v7.i10.1377

Li, Z. H., Tang, Q. B., Wang, J., Zhou, L., Huang, W. L., Liu, R. Y., et al. (2010). Hepatitis $\mathrm{C}$ virus core protein induces malignant transformation of biliary epithelial cells by activating nuclear factor-kappaB pathway. J. Gastroenterol. Hepatol. 25, 1315-1320. doi: 10.1111/j.1440-1746.2009.06201.x

Mahale, P., Torres, H. A., Kramer, J. R., Hwang, L. Y., Li, R., Brown, E. L., et al. (2017). Hepatitis C virus infection and the risk of cancer among elderly US adults: a registry-based case-control study. Cancer 123, 1202-1211. doi: 10. 1002/cncr.30559

Mao, Y., Chen, W., Wu, H., Liu, C., Zhang, J., and Chen, S. (2020). Mechanisms and functions of MiR-200 family in hepatocellular carcinoma. Onco Targets Ther. 13, 13479-13490. doi: 10.2147/OTT.S288791

Mayakonda, A., Lin, D. C., Assenov, Y., Plass, C., and Koeffler, H. P. (2018). Maftools: efficient and comprehensive analysis of somatic variants in cancer. Genome Res. 28, 1747-1756. doi: 10.1101/gr.239244.118

Merte, B. (1989). [The first general mathematical description of optical lenses without aberrations]. Klin. Monbl. Augenheilkd. 194, 59-61.

Mohsen, M. A. A., Hussein, N. A., Ghazal, A. A., El-Ghandour, M. K., Farouk, M., El-Wahab, A., et al. (2014). Angiogenic output in viral hepatitis, C and B, and HCV-associated hepatocellular carcinoma. Alexandria J. Med. 50, 235-240.

Montenegro, L., De Michina, A., Misciagna, G., Guerra, V., and Di Leo, A. (2013). Virus $\mathrm{C}$ hepatitis and type 2 diabetes: a cohort study in southern Italy. Am. J. Gastroenterol. 108, 1108-1111. doi: 10.1038/ajg.2013.90

Naggie, S. (2017). Hepatitis C virus, inflammation, and cellular aging: turning back time. Top. Antivir. Med. 25, 3-6.

Ninio, L., Nissani, A., Meirson, T., Domovitz, T., Genna, A., Twafra, S., et al. (2019). Hepatitis C virus enhances the invasiveness of hepatocellular carcinoma 
via EGFR-mediated invadopodia formation and activation. Cells 8:1395. doi: $10.3390 /$ cells8111395

Ozkok, A., and Yildiz, A. (2014). Hepatitis C virus associated glomerulopathies. World J. Gastroenterol. 20, 7544-7554. doi: 10.3748/wjg.v20.i24.7544

Parkin, D. M. (2006). The global health burden of infection-associated cancers in the year 2002. Int. J. Cancer 118, 3030-3044. doi: 10.1002/ijc.21731

Ramachandran, S., Ilias Basha, H., Sarma, N. J., Lin, Y., Crippin, J. S., Chapman, W. C., et al. (2013). Hepatitis C virus induced miR200c down modulates FAP-1, a negative regulator of Src signaling and promotes hepatic fibrosis. PLoS One 8:e70744. doi: 10.1371/journal.pone.0070744

Sato, Y., Nakata, K., Kato, Y., Shima, M., Ishii, N., Koji, T., et al. (1993). Early recognition of hepatocellular carcinoma based on altered profiles of alphafetoprotein. N. Engl. J. Med. 328, 1802-1806. doi: 10.1056/NEJM19930624328 2502

Shiu, T. Y., Shih, Y. L., Feng, A. C., Lin, H. H., Huang, S. M., Huang, T. Y., et al. (2017). HCV core inhibits hepatocellular carcinoma cell replicative senescence through downregulating microRNA-138 expression. J. Mol. Med. 95, 629-639. doi: 10.1007/s00109-017-1518-4

Tholey, D. M., and Ahn, J. (2015). Impact of hepatitis C virus infection on hepatocellular carcinoma. Gastroenterol. Clin. North Am. 44, 761-773. doi: 10.1016/j.gtc.2015.07.005

Tong, Z., Cui, Q., Wang, J., and Zhou, Y. (2019). TransmiR v2.0: an updated transcription factor-microRNA regulation database. Nucleic Acids Res. 47, D253-D258. doi: 10.1093/nar/gky1023

Umezu, T., Tsuneyama, K., Kanekura, K., Hayakawa, M., Tanahashi, T., Kawano, M., et al. (2020). Comprehensive analysis of liver and blood miRNA in precancerous conditions. Sci. Rep. 10:21766. doi: 10.1038/s41598-020-78500-1

Vescovo, T., Refolo, G., Vitagliano, G., Fimia, G. M., and Piacentini, M. (2016). Molecular mechanisms of hepatitis $\mathrm{C}$ virus-induced hepatocellular carcinoma. Clin. Microbiol. Infect. 22, 853-861. doi: 10.1016/j.cmi.2016.07.019

Vlachos, I. S., Paraskevopoulou, M. D., Karagkouni, D., Georgakilas, G., Vergoulis, T., Kanellos, I., et al. (2015). DIANA-TarBase v7.0: indexing more than half a million experimentally supported miRNA:mRNA interactions. Nucleic Acids Res. 43, D153-D159. doi: 10.1093/nar/gku1215

Wang, S. C., Li, C. Y., Chang, W. T., Cheng, W. C., Yen, C. H., Tu, W. Y., et al. (2021). Exosome-derived differentiation antagonizing non-protein coding RNA with risk of hepatitis $\mathrm{C}$ virus-related hepatocellular carcinoma recurrence. Liver Int. 41, 956-968. doi: 10.1111/liv.14772

Welchew, D. E., Honey, G. D., Sharma, T., Robbins, T. W., and Bullmore, E. T. (2002). Multidimensional scaling of integrated neurocognitive function and schizophrenia as a disconnexion disorder. Neuroimage 17, 1227-1239. doi: 10.1006/nimg.2002.1246

Weng, G., Bhalla, U. S., and Iyengar, R. (1999). Complexity in biological signaling systems. Science 284, 92-96. doi: 10.1126/science.284.5411.92

Wong, C. M., Tsang, F. H., and Ng, I. O. (2018). Non-coding RNAs in hepatocellular carcinoma: molecular functions and pathological implications. Nat. Rev. Gastroenterol. Hepatol. 15, 137-151. doi: 10.1038/nrgastro.2 017.169

Zhang, W., Gao, Y., Li, F., Tong, X., Ren, Y., Han, X., et al. (2015). YAP promotes malignant progression of Lkbl-deficient lung adenocarcinoma through downstream regulation of survivin. Cancer Res. 75, 4450-4457. doi: 10.1158/0008-5472.CAN-14-3396

Zhang, W., Nandakumar, N., Shi, Y., Manzano, M., Smith, A., Graham, G., et al. (2014). Downstream of mutant KRAS, the transcription regulator YAP is essential for neoplastic progression to pancreatic ductal adenocarcinoma. Sci. Signal. 7:ra42. doi: 10.1126/scisignal.2005049

Zheng, Y. B., Huang, J. W., Zhan, M. X., Zhao, W., Liu, B., He, X., et al. (2014). Genetic variants in the KDR gene is associated with the prognosis of transarterial chemoembolization treated hepatocellular carcinoma. Tumour Biol. 35, 11473-11481. doi: 10.1007/s13277-014-2478-8

Zhou, Q., Lui, V. W., and Yeo, W. (2011). Targeting the PI3K/Akt/mTOR pathway in hepatocellular carcinoma. Future Oncol. 7, 1149-1167. doi: 10.2217/fon. 11.95

Conflict of Interest: The authors declare that the research was conducted in the absence of any commercial or financial relationships that could be construed as a potential conflict of interest.

Publisher's Note: All claims expressed in this article are solely those of the authors and do not necessarily represent those of their affiliated organizations, or those of the publisher, the editors and the reviewers. Any product that may be evaluated in this article, or claim that may be made by its manufacturer, is not guaranteed or endorsed by the publisher.

Copyright (c) 2021 Chen, Zhang and Ben. This is an open-access article distributed under the terms of the Creative Commons Attribution License (CC BY). The use, distribution or reproduction in other forums is permitted, provided the original author(s) and the copyright owner(s) are credited and that the original publication in this journal is cited, in accordance with accepted academic practice. No use, distribution or reproduction is permitted which does not comply with these terms. 